\title{
Modeling and Analysis of Reliability in Grid using Petri Nets
}

\author{
Mahnoosh Vahebi \\ Department of Computer \\ Science and Research Branch \\ Islamic Azad University, \\ Gilan. Iran
}

\author{
Mehdi Sadeghzadeh \\ Department of Computer \\ Science and Research Branch \\ Islamic Azad University \\ Mahshahr, Iran
}

\author{
Reza Ebrahimi Atani \\ Department of Science and \\ Research Branch, \\ Islamic Azad University, \\ Gilan. Iran
}

\begin{abstract}
Intelligent Grid computing is considered as an essential infrastructure in future life, and they are executed and implemented to save the energy, to reduce the costs and to increase reliability. This study has been carried out with the aim of identifying various samples of Grid networks and investigating the applied and functional fields. In this way, science and technology develops and expands through identifying their weaknesses and attempting to remove these weaknesses and to solve the problems. In addition, the purpose is to increase the reliability in grid networks for using the services. In fact, the purpose of this study is to provide and present a compact and intensive mathematical tool by using Petri nets in order to analyze reliability and certainty in Grid networks. In this study, reliability is investigated in Grid services, and through using Petri nets, a model is represented and proposed for computing as well as increasing reliability in Grid networks. In this research, a new model has been introduced for increasing reliability in Grid networks. In this model, by using series-parallel mode and also by adding redundancy technique to the system, reliability has been considerably improved.
\end{abstract}

Keywords: grid network- petri net- Reliability- cpn tools- Resource managment

\section{INTRODUCTION}

Nowadays, scientific issues and problems are very complicated and require great storage spaces and high computational power. Traditional techniques such as parallel and distributive computing are not appropriate methods for solving such problems. Therefore, Grid networks are proposed in late $90 \mathrm{~s}$, and nowadays, they are widely used in developed countries with the aim of preventing waste of energy and using them optimally.

Grid computing is considered as a software and hardware infrastructure, and allows us to access high computational capabilities in a certain, compatible, cheap and broad way. Grid computing is related to a set of heterogeneous resources in large scales such as personal computers, work stations of clusters and supercomputers. These resources vary in terms of computational capabilities and configuration, and they are organized according to different management policies. Grid computing environment is suitable and proper bed for solving the problems requiring long and difficult computing.

In software or a set of software use grid, some issues including information subscription, information dividing, its transferring by considering full security and management of information will be very difficult and important.

The most important issue and problem mentioned in this paper is resource subscription. In Grid, resource subscription is controlled by management system of control resources. The request sent by user to RMS is called task.RMS should divide the received task into sub-tasks, and should send them to resources available in the network. In order to reach the purpose of Grid and to use resources available in Grid environment, scheduling and distribution of sub-tasks among resources are performed by considering the quality of service. In the procedure of scheduling, sub-tasks should be distributed between resources in a way that maximum QOS is produced.
Service reliability in Grid environment should be studies as one of the most significant QOS criteria. Execution time is a duty and is considered as a factor for computing reliability in Grid environment. Always, it has been tried to perform a task in the time lesser than determined time. Usually, technique used in Grid environment to increase reliability is redundancy technique. After dividing tasks into sub-tasks, RMS does not assign each task to one resource; rather, it assigns it to several resources so that all of them process the related sub-task simultaneously. Also, if one of these resources is destroyed, other resources try to compensate it.

\section{LITERATURE REVIEW}

Azgami in [10] , has considered modeling of tasks distribution and reliability computing in Grid networks. In this paper, management system of resources has undertaken the task basis of Grid networks. This system receives the tasks from users, and divides them into sub-tasks. Then, it sends each sub-task to several available resources. Finally, after executing them by connected resources, outputs are collected, and user's request task is responded. Above mentioned operations are simulated by time colored Petri nets, and reliability is computed.

Zeng in [1], has analyzed reliability in control center networks in smart Grid using stochastic Petri nets. In his article, the purpose is to present a compact and intensive mathematical tool by stochastic Petri nets (SPNs) to analyze reliability in control center network in smart Grid network.

In this paper, a method has been presented to repair the unit server that has been destroyed. Then, two supporting strategies in critical servers have been studies and investigated, and SPNs have been presented. Finally, above mentioned model combines with general model of the purpose of control center network.

In this paper, in control center, we have considered a network consisting of a control center and $\mathrm{N}$ sub-network. In control center, SCADA server, data base and application servers are 
connected to local networks (LAN) supported by profile. One a section fails or is exposed to different attacks in control center, then it can be immediately repaired by user interface. In addition, supportive services are used in order to improve reliability.

Dastgheibi Fard, in[11]: has presented a new scheduler algorithm for grid computing. He has firstly introduced LMB algorithm. Then, weaknesses of this algorithm have been mentioned, and NLMB algorithm has been proposed and introduced. The purpose of this algorithm is to reduce removed deadlines, to increase sustainability of errors and to reduce return time. The proposed algorithm considers more parameters in scheduling in comparison with that latest presented algorithm, and reduces removed deadlines to a considerable degree. Also it increases sustainability of error.

\section{PROPOSE ALGORITHM}

In proposed model, following hypothesis have been taken into account:

1) In Grid environment, star topology has been used and applied, and RMS is connected to all resources. Communication lines are unique between each resource and RMS.

2) Users using Grid send their own requests to RMS. Then, RMS divides them into sub-tasks, and sends them to resources.

3) Sub-tasks do not depend on each other. As soon as resources receive sub-task data from RMS, they begin to execute it.

4) RMS is very quick and reliable, so the time of task processing by RMS can be ignored in comparison with the time of sub-task processing.

5) Grid environment reliability refers to the probability of presenting the results of a task in the time lesser than determined time, $\mathrm{T}$ (reliable).

In the proposed algorithm, redundancy technique is used to increase reliability. In this algorithm, one sub0task is allocated to several resources on the basis of redundancy technique. In previous algorithm, resources are provided for sub-tasks stochastically, while in proposed method, each sub-task considers the most appropriate resources on the basis of data required to execute each sub-task and its complex computing as well as bandwidth of resources and processing speed of each resource (see figure1 of the model).

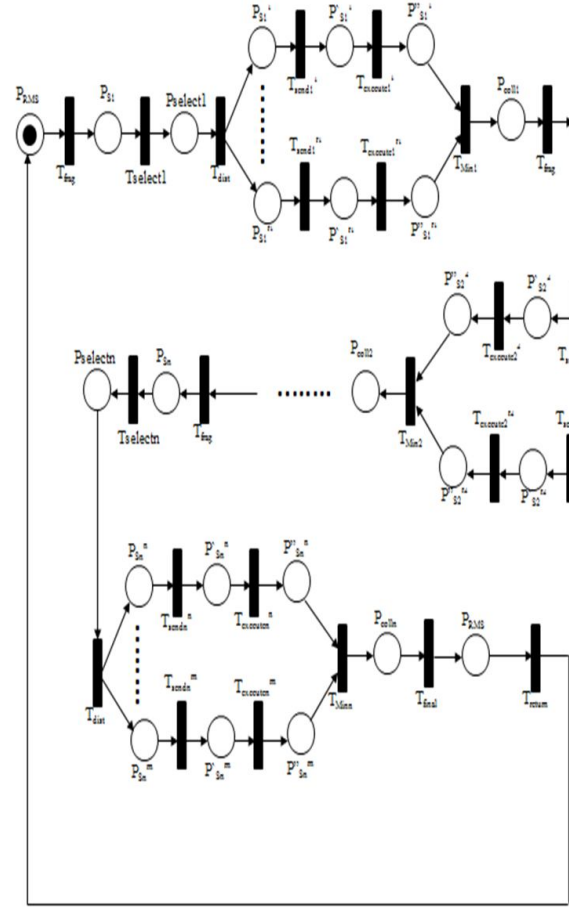

Figure 1. Proposed Model

Let's compute reliability as follows:

Data required executing $S_{i}$ sub-task that should be sent from RMS to $R_{j}$ resource is shown by $\operatorname{rd}_{i}$. The results that should be sent from $R_{j}$ resource to RMS are demonstrated by $I_{i}$. Data transferred and sent to execute $S_{i}$ sub-task between RMS and $\mathrm{R}_{\mathrm{j}}$ resource can be shown by $\mathrm{a}_{\mathrm{i}}$, and it is computed as follows:

$\mathrm{A}_{\mathrm{i}}=\mathrm{rd}_{\mathrm{i}}+\mathrm{I}_{\mathrm{i}}$

If bandwidth of communication lines between RMS and $\mathrm{R}_{\mathrm{j}}$ resource is demonstrated by $b_{\mathrm{j}}$, then transferred time is computed as follows:

$\mathrm{T}_{\mathrm{ij}}=\mathrm{a}_{\mathrm{i}} / \mathrm{bw}_{\mathrm{j}}$

Transferred and sent time adds transferred time to tokens transferred from $\mathrm{P}^{\mathrm{j}}$ location to $\mathrm{P}^{\mathrm{i}}$ location. In addition, when tokens pass through transferring $\mathrm{T}$ (send), this issue should be considered that tokens should not face with failing during transferring data $\left(\mathrm{q}_{\mathrm{ij}}\right)$. Therefore, when tokens pass through transferring, a pair of $\left(\mathrm{T}_{\mathrm{ij}}, \mathrm{q}_{\mathrm{ij}}\right)$ is added to them. If computing complexity of all entered tasks is shown by c, then computational complexity of each sub-task is computed according to the following equation:

$\sum_{i=1}^{n} C_{i}=C$

In this equation, $\mathrm{n}$ indicates the number of sub-tasks related to a specific task, and $c_{i}$ is computational complexity of each $s_{i}$ task. If processing speed of $R_{j}$ is shown by $P_{s j}$, then processing time of $\mathrm{s}_{\mathrm{i}}$ sub-task is obtained through the following equation:

$T_{i j}=C_{i} / p s_{j}$

As it is observed in figure, time transferring of T(execute) can add processing time of sub-task $\left(\mathrm{T}_{\mathrm{ij}}\right)$ to time of data transferring $\left(\mathrm{t}_{\mathrm{ij}}\right)$, and adds the obtained result to token. In 
addition, the probability of lack of failing during sub-task $\left(\mathrm{P}_{\mathrm{ij}}\right)$ processing is multiplied by the probability of lack of failing during data transferring $\left(\mathrm{q}_{\mathrm{ij}}\right)$, and is added to token. Hence, tokens passing from transferring $\mathrm{T}$ (execute), it carries a pair of $\left(t_{i j} * T_{i j}\right),\left(p_{i j}, q_{i j}\right)$ that is an indication of successful execution time and its probability. When redundancy technique is used to increase reliability, each sub-task is processed by several processing resources in simulation. Therefore, $\mathrm{T}_{\mathrm{iRi}}$ is an indication of minimum execution time of $s_{i}$ sub-task in resources, and it is obtained by the following equation:

$T_{i, R r i}=\min \left(t_{i j}+T_{i j}\right) ; \quad k \in R_{r i}$

With regard to above mentioned equation as well as a pair of $\left(\mathrm{t}_{\mathrm{ij}} * \mathrm{~T}_{\mathrm{ij}}\right),\left(\mathrm{p}_{\mathrm{i} j}, \mathrm{q}_{\mathrm{ij}}\right)$, the minimum execution time of $\mathrm{s}_{\mathrm{i}}$ sub-task and probability of $\left(\mathrm{P}_{\mathrm{i}, \mathrm{Rr}}\right)$ have been placed over token after transferring through $\mathrm{T}(\mathrm{min})$ passage, and it is located in $\mathrm{P}($ coll $)$ place. In order to end a task, all sub-tasks should be executed. Hence, when tokens are placed over all $\mathrm{P}$ (coll) places, $\mathrm{T}$ (final) transferring begins, and it considers the greatest execution time of sub-task as execution time of task. It is obtained through the following equation:

$T=\sum_{i=1}^{n} T_{i}$

In this equation, $\mathrm{n}$ indicates the number of sub-tasks related to each sub-task. In other words, in order to execute a task, this task is firstly divided into sub-tasks. Afterwards, completion time is computed in each resource and for each sub-task. Minimum completion time is determined in each source and for each sub-task. After performing this process for all subtasks, minimum time is selected among the least completion time of resources for execution.

When tokens pass through $\mathrm{T}(\max )$ transferring, and are placed in $\mathrm{P}(\mathrm{RMS} 2)$ location, the operations of task execution come to end. By beginning $\mathrm{T}$ (return), another task can enter the system.

The following equation is used to compute reliability:

$R_{T^{*}}=\prod_{i=1}^{n} P_{i} * l\left(T<T^{*}\right)$

In this equation, I indicates total number of $t . p_{i}$ is the probability of executing a task in $\mathrm{T}_{\mathrm{i}}$ time, and $\mathrm{T}^{*}$ is $\mathrm{T}_{\text {reliable }}$ time. Also, 1 function is defined as follows:

$$
l(\text { true })=1 \quad, \quad l(\text { false })=0
$$

In addition, the following equation is used to compute $\mathrm{p}_{\mathrm{i}}$ reliability:

$P_{i}=1-\prod_{J=1}^{n}\left(1-R_{j}\right)$

\section{CASE STUDY}

Let's implement our proposed algorithm in a system, and measure reliability and response time according to mentioned methods.

In case studies, we try to present comprehensive and small samples. In this way, the samples are not very complicated. Also we try to present a small sample which is representative of real and large samples. For example, ATM system has been considered with a properC4ISR architectural structure because it is not very complex, and creating its architectural structure is possible. Then, the problem is explained, and an executable mode for architectural products (UML diagrams) is created. Afterwards, through using the proposed method and simulation of the model that can be used and executed in CPN tools, reliability and response time are computed. ATM interacts with user and bank. In this system, one of the main user operations is withdrawing money from the account. The following figure shows is withdrawing money from the account (see figure 2).

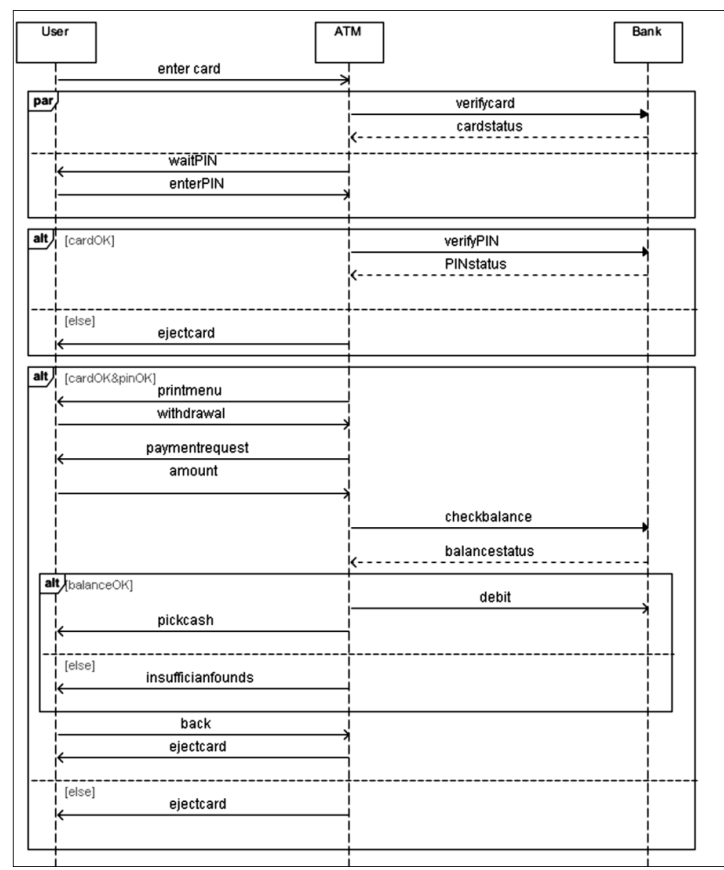

Figure 2. ATM system

\subsection{Simulation}

With regard to the model presented in chapter of proposed algorithm, ATM system shown in previous section is simulated with regard to explanations of proposed algorithm, primary characteristics of system should be firstly determined. These characteristics involve bandwidth of system, computational complexity for each job which has been entered the system, and data required for each job and work. After determining above mentioned issues and factors, system is simulated. ATM system has been implemented in CAPTOOLS simulator software as follows (see figure 3):

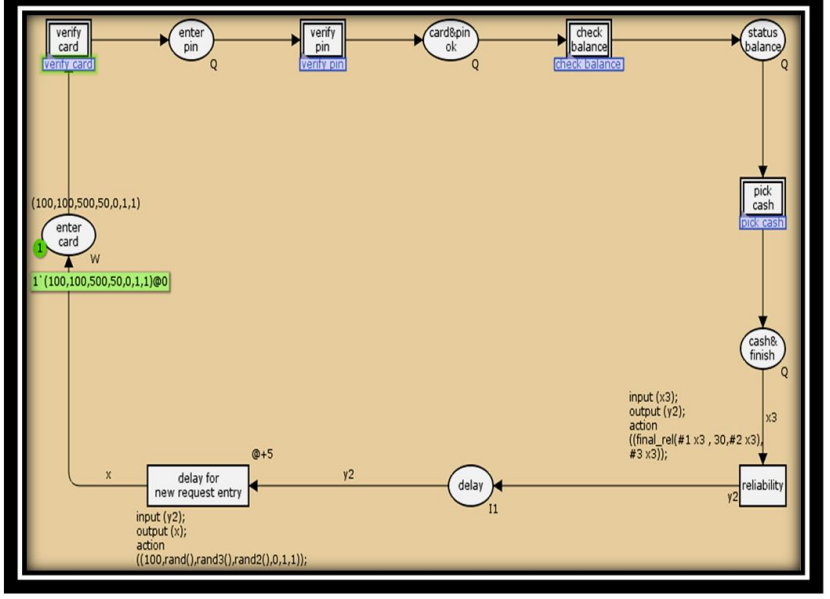

Figure 3.ATM simulation system by cpn tools 
Let's consider subsystems of ATM system. Hence, there are four sub-systems as follows: card entry and investigating it, entering card password, selection of request type (deposit request), receiving money. In this paper, just one subsystem is explained in details. These subsystems have been implemented in CPNTOOLS simulator as follows (see figure 4):

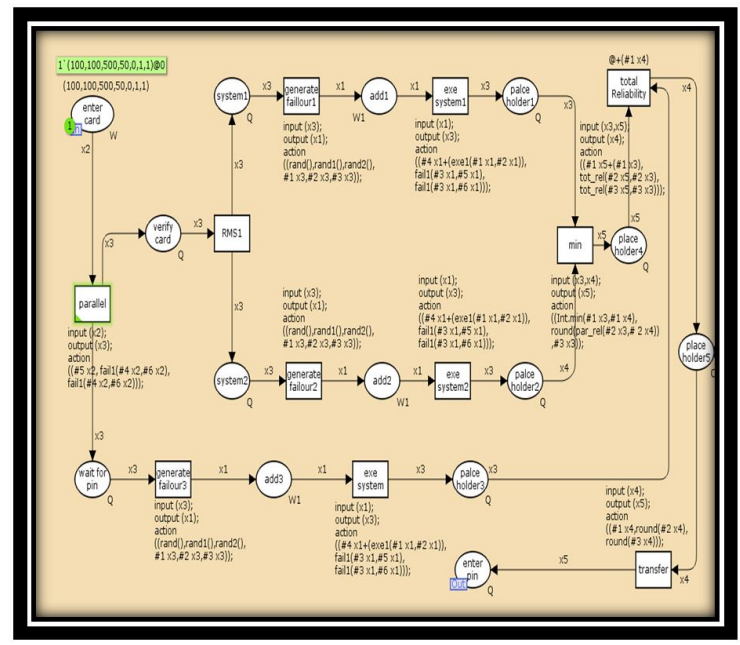

Figure 4. Subsystem of ATM System

After simulating the system, we compare the results obtained from system with parallel systems (Azgami model) and series system (normal mode). The following figure shows this comparison (see figure 5). According to diagram, it's obvious that our proposed model improves reliability to a considerable degree.

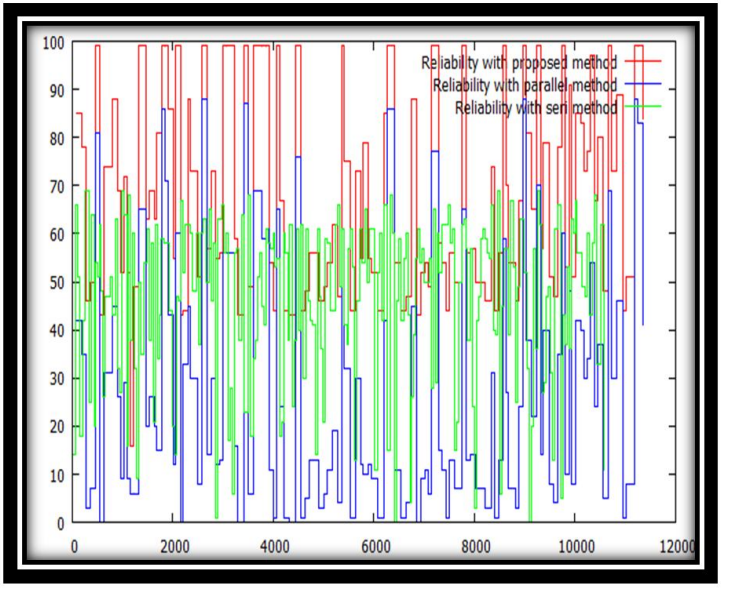

Figure 5. Simulation Diagram

\section{CONCLUSION}

In this paper, the proposed algorithm has considerably improved reliability through adding redundancy technique to system and using series-parallel modes. Since colored Petri nets involve powerful mathematical power in terms of evaluating software systems, we use VCPNTOOLS simulator to simulate the proposed model. Also, we compare our model with two other models (series and parallel models). The results obviously show that the proposed algorithm and model is preferred to other algorithms that have been proposed up to now.

\section{SUGGESTIONS}

Since no work is complete and there are certainly some faults in the work and job, the model presented in this paper has some faults. This issue can be considered as a background for performing great projects. With regard to proper application of Petri nets in improving systems, an optimum model has been proposed in this paper to increase reliability in Grid. Comparing this method with previous methods show improvement of efficiency and increasing accessibility.

In this paper, the following suggestions have been proposed:

1) Deleting some hypothesis of the problem and involving more real hypothesis and suggestions for problem solving.

2) Using above mentioned model and generalization of this method for other Grid topologies such as hierarchical and tree topology.

3) Considering data dependency among subtasks and expecting some sub-tasks to receive required data from sub-tasks.

\section{REFERENCES}

[1] Rongfei Zeng,Yixin Jiang,Chuang Lin, and Xuemin (Sherman) Shen "Dependability Analysis of Control Center Networks in Smart Grid using Stochastic Petri Nets" Department of Electrical and Computer Engineering Sept. 2012 (vol. 23 no. 9) pp. 1721-1730.

[2] Gholami Ehsan; Rahmani Amir Masoud; Habibizad navin Ahmad;" Using Monte Carlo Simulation in Grid Computing Systems for Reliability Estimation"; 2009 Eighth International Conference on Networks. PAGE: 380-384 E- ISBN 978-0-7695-3552-4.

[3] Tanenbaum As., Vansteen M.2007, Distributed Systems .Principles and paradigms.Prentice Hall,2007. iSBN 013-239227-5.

[4] Allan Mazur*, Todd Metcalfe;" America's three electric grids: Are efficiency and reliability functions of grid size"; Electric Power Systems Research 89 (2012) 191195. VOLUME 89 Elsevier.

[5] Mohammad Khanli Leyli, Etminan Far Maryam, Ghaffari Ali.," Reliable Job Scheduler using RFOH in Grid Computing", Journal of Emerging Trends in Computing and Information Sciences, (02009-2010 CIS Journal. All rights reserved. VOL. 1, NO. 1, JULY 2010 ISSN 2079-8407.

[6] Parsa Saeed , Azadi Parand Fereshteh-.," Estimation of service reliability and performance in grid environment, Journal of King Saud University - Engineering Sciences (2012) 24, 151-157. July 2012.

[7] Y.S. Dai, M. Xie, K.L. Poh.," Reliability Analysis of Grid Computing Systems", Department of Industrial and System Engineering, National University of Singapore. ISBN: 0-306-48496-X.vol. 79, no. 1, pp. 103-112.

[8] Hiroyuki Mori, Hiroki Kakuta" Modified SPEA2 for Probabilistic Reliability Assessment in Smart Grids" , Procedia Computer Science 6 (2011)..

[9] Abawajy j.2004.placement of filereplicas in data environments:lecture Note in computer science (LNCS).springer,heidelborg.3038:66-73.

[10] Azgomi mohammad abdollahi,entezari maleki reza, esfand $87 "$ Reliability modeling distribution of tasks and 
International Journal of Computer Applications Technology and Research

Volume 2- Issue 6, 726 - 730, 2013, ISSN: 2319-8656

calculate a star topology grid services using colored Petri Nets Time".

[11] Dastgheybifar Gholamhoseyn, Ansari Sara, Lotfi Somayeh ,1386 " A new scheduling algorithm for computational grid" Thirteenth Annual Conference of Computer Society of Iran
[11] Naseri a, Rahmani a , Jola, Esfand 1389 " New method for optimizing the time - cost of resource allocation in grid system, and implemented it using gravitational search algorithm" First National Conference on Soft Computing and Information Technology. 